\title{
A novel unidirectional porous $\beta$-tricalcium phosphate grafting for vertebral fracture in the elderly: preliminary case series
} \author{
Tetsuya Abe' ${ }^{1}$, Kousei Miura1', Katsuya Nagashima', Kentaro Mataki ${ }^{1}$, Yosuke Shibao', \\ Kosuke Sato ${ }^{1}$, Masao Koda ${ }^{1}$, and Masashi Yamazaki ${ }^{1}$ \\ ${ }^{I}$ Department of Orthopedic Surgery, Faculty of Medicine, University of Tsukuba, Japan \\ ${ }^{2}$ Department of Orthopedic Surgery, Kenpoku Medical Center Takahagi Kyodo Hospital, Japan
}

Toru Funayama1, Toshinori Tsukanishi², Hiroshi Kumagai ${ }^{1}$, Hiroshi Noguchi ${ }^{1}$, Shigeo Izawa ${ }^{1}$,

\begin{abstract}
Objective: To treat vertebral fractures with posterior wall injury in the elderly, vertebral bone grafting is generally performed through a posterior transpedicular approach, combined with pedicle screw fixation. An autologous bone is ideal to treat this disorder. However, harvesting autologous bones from the elderly with osteoporosis is limited by the amount and quality of available autologous bone. Thus, we developed a bone-grafting substitute. The newly developed unidirectional porous $\beta$-tricalcium phosphate, with a porosity of $57 \%$ (UDPTCP; Affinos ${ }^{\circledR}$, Kuraray Co., Ltd., Tokyo, Japan), is used in the bone-grafting procedure. This is the first report of UDPTCP used as an artificial bone graft in patients with an acute vertebral burst fracture.

Materials and Methods: UDPTCP (mean: $4.2 \mathrm{~g}$ ) was implanted through the pedicle, and posterior instrumentation was achieved with pedicle screws in five elderly patients. Resorption of UDPTCP and substitution with the autologous bone were evaluated on computed tomography (CT) and plain X-ray performed immediately and at 3, 6, and 12 months after the operation.

Results: In case 1, the pedicle screws did not loosen, and UDPTCP was completely resorbed and replaced with the autologous bone at 3 postoperative months. In the other four cases, although the pedicle screws or the caudal part loosened because of osteoporosis, resorption of UDPTCP was observed at 3 postoperative months. At 6 postoperative months, progressive substitution with the autologous bone was confirmed, and at 12 postoperative months, formation of the good autologous bone was confirmed.

Conclusion: This preliminary case series demonstrated that the newly developed UDPTCP shows good clinical potential as a bonegraft substitute for acute vertebral burst fractures in the elderly, including patients with osteoporosis.
\end{abstract}

Key words: artificial bone, unidirectional porous $\beta$-tricalcium phosphate, vertebral fracture, elderly patients, osteoporosis

\section{Introduction}

Bone grafting is often performed to treat the bone fractures that are accompanied with a bone defect. An autogenous bone graft is optimal, but it has drawbacks in the pro-

Received: April 21, 2019

Accepted: June 28, 2019

Correspondence: Toru Funayama, Department of Orthopedic Surgery, Faculty of Medicine, University of Tsukuba, 1-1-1 Tennodai, Tsukuba, Ibaraki 305-8576, Japan

E-mail: funatoru3@md.tsukuba.ac.jp

This is an open-access article distributed under the terms of the Creative Commons Attribution Non-Commercial No Derivatives
(by-nc-nd) License $<$ http://creativecommons.org/ licenses/by-nc-nd/4.0/>. cedures and quantity of bone graft, especially in the elderly patients with osteoporosis. Therefore, various types of artificial bones have been developed and clinically used. Unidirectional porous $\beta$-tricalcium phosphate (UDPTCP; Affi$\operatorname{nos}^{\circledR}$, Kuraray Co., Ltd., Tokyo, Japan) is a novel bone-graft substitute, with a porosity of $57 \%$, containing pores with a diameter of 25-300 $\mu \mathrm{m}$ in one direction ${ }^{1}$. Animal experiments showed that the the artificial bone with unidirectional pores is characterized by good balance between its resorption and substitution with the autologous bone ${ }^{2,3)}$. However, only a few clinical studies have evaluated its implantation characteristics in humans ${ }^{4-7)}$. This study is the first report of UDPTCP used for artificial bone grafting in the vertebrae. 


\section{Materials and Methods}

The Institutional Review Board approval and consent to participate from patients was obtained. The trial registration number of Kenpoku Medical Center Takahagi Kyodo Hospital is TKH-IRB2015-001. Written informed consent was obtained from the five patients for publication of the paper and accompanying images.

\section{Case summary (Table 1)}

Case 1

A 66-year-old woman with back pain was admitted to our hospital after a minor traffic accident. Radiographic imaging revealed an acute burst fracture in the L1 vertebra. The patient had no history of osteoporosis. However, the young adult mean (YAM) of bone density was 69\%. Two grams of UDPTCP granules were implanted in the defective bone area, and five vertebrae (T11 to L3) were fused with the pedicle screws (PS).

Case 2

A 67-year-old woman with back pain was admitted to our hospital after a fall and loss of consciousness. Radiographic imaging revealed an acute burst fracture in the T12 vertebra. The patient had no history of osteoporosis. However, the YAM of bone density was 58\%. Four grams of UDPTCP granules were injected in the defective bone area, and five vertebrae (T10 to L2) were fused with PS.

Case 3

A 73-year-old woman with back pain was admitted to our hospital after she had fallen on her back. Radiographic imaging revealed a fracture in the L1 vertebra. The patient had a history of osteoporosis, and the YAM of bone density was $70 \%$. Six grams of UDPTCP granules were implanted in the defective bone area, and six vertebrae (T10 to L3) were fused with PS.

Case 4

A 77-year-old woman complaining of back pain was admitted to our hospital after she had twisted her trunk. Radiographic imaging revealed an acute burst fracture of the L1 vertebra. The patient had a history of osteoporosis, and the YAM of bone density was $76 \%$. Five grams of UDPTCP granules were injected in the defective bone area, and six vertebrae (T10 to L3) were fused with PS.

Case 5

An 86-year-old woman with back pain was admitted to our hospital after she experienced a fall. Radiographic imaging revealed a fracture in the T12 vertebra. The patient had no history of osteoporosis. However, the YAM was $71 \%$. Four grams of UDPTCP granules were injected in the defective bone area, and seven vertebrae (T9 to L3) were fused with PS.

\section{Surgical procedures}

Bone grafting was performed by injecting a sufficient amount of UDPTCP granules (mean $4.2 \mathrm{~g}$ ) in the defective part of the vertebral body using a percutaneous vertebroplasty device (Asro Medical, Ibaraki, Japan) through the pedicle. During implantation, excessive compression was avoided to preserve the porous structure. Moreover, posterior instrumentation was added by fluoroscopically inserting PS into the 2-3 vertebrae cranial and 2-3 vertebrae caudal to the affected vertebra ${ }^{8)}$. Postoperatively, teriparatide administration was started in all patients to treat osteoporosis.

\section{Outcome measurements}

Resorption of UDPTCP, substitution by the autologous bone, and loosening of PS, which was seen as a radiolucent area around PS, were evaluated on computed tomography (CT) and plain X-ray performed immediately and at 3, 6, and 12 months after operation. The CT-value (Hounsfield units) was used to monitor changes in the anterior part of the vertebra, in which UDPTCP was filled, and in the posterior part of the vertebra, in which the burst bone was located (Figure 1). The CT-value was calculated using the Osirix software (Pixmeo SARL, Geneva, Switzerland) after the regions of interest had been set based on previous studies ${ }^{9,10)}$.

\section{Results}

In case 1 , PS did not loosen, and at 3 postoperative

Table 1 Summary of patient demographic information

\begin{tabular}{cccccccccc}
\hline Case & Age & Sex & $\begin{array}{c}\text { Height } \\
(\mathrm{cm})\end{array}$ & $\begin{array}{c}\text { Body weight } \\
(\mathrm{kg})\end{array}$ & $\begin{array}{c}\text { Spinal level of } \\
\text { the fracture }\end{array}$ & $\begin{array}{c}\text { YAM } \\
(\%)\end{array}$ & $\begin{array}{c}\text { Operated spinal } \\
\text { levels (number) }\end{array}$ & $\begin{array}{c}\text { Screws } \\
\text { (Company) }\end{array}$ & $\begin{array}{c}\text { Amount of } \\
\text { UDPTCP (g) }\end{array}$ \\
\hline 1 & 66 & F & 145 & 45.0 & L1 & 69 & T11-L3 (5) & ES2 (Stryker) & 2 \\
2 & 67 & F & 153 & 48.7 & T12 & 58 & T10-L2 (5) & Voyager (Medtronic) & 4 \\
3 & 73 & F & 143 & 62.0 & L1 & 70 & T10-L3 (6) & IBIS (Japan MDM) & 6 \\
4 & 77 & F & 157 & 62.7 & L1 & 76 & T10-L3 (6) & ES2 (Stryker) & 5 \\
5 & 86 & F & 150 & 51.0 & T12 & 71 & T9-L3 (7) & IBIS (Japan MDM) & 4 \\
\hline mean & 73.8 & - & 149.6 & 53.9 & - & 68.8 & $(5.8)$ & - & 4.2 \\
\hline
\end{tabular}

YAM: young adult mean; UDPTCP: unidirectional porous $\beta$-tricalcium phosphate (Affinos ${ }^{\circledR}$ ). 


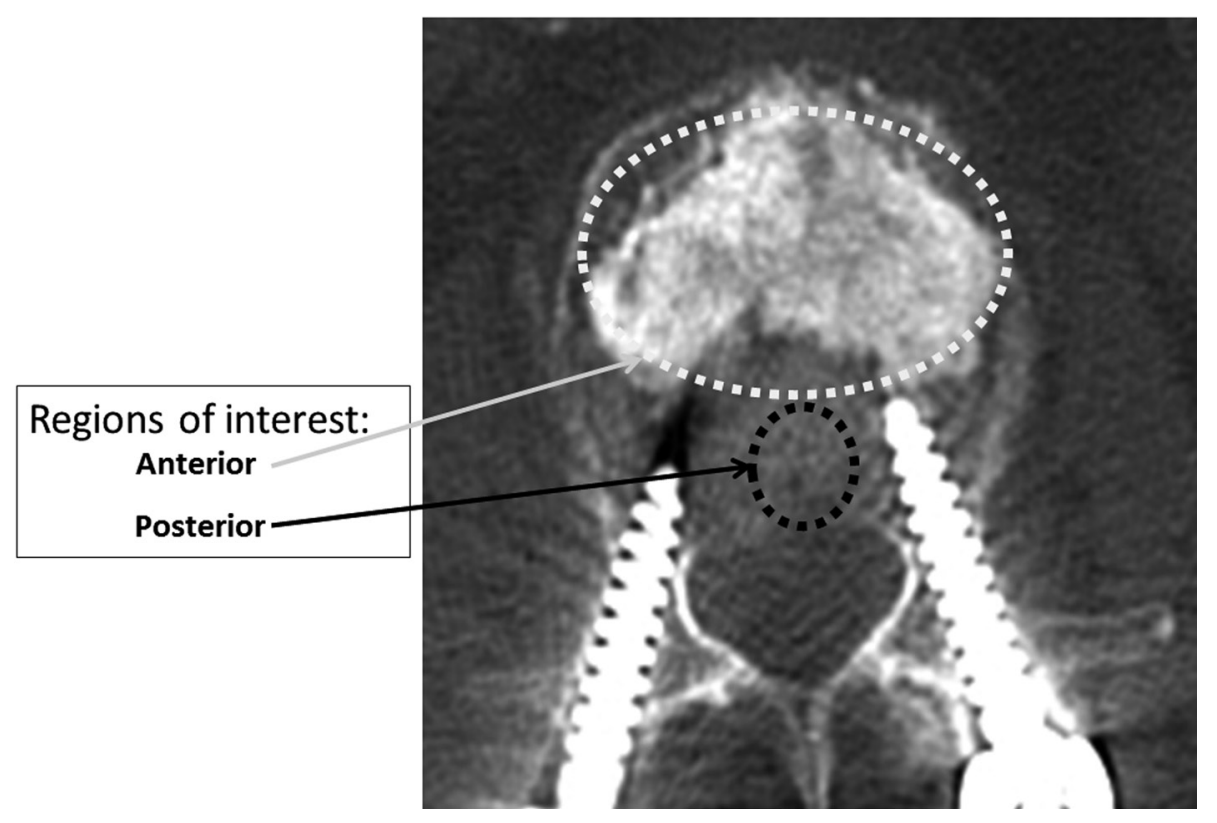

Figure 1 Computed tomography (CT)-value calculation.

The CT-value (Hounsfield units) was used to monitor changes in the anterior part of the vertebra, in which unidirectional porous $\beta$-tricalcium phosphate (UDPTCP) was filled, and in the posterior part of the vertebra, in which the burst bone was located. The CT-value was calculated after the regions of interest had been set based on previous studies ${ }^{9,10)}$.

Table 2 The computed tomography (CT)-value (Hounsfield units) in patients after operation for an acute vertebral burst fracture

\begin{tabular}{ccccc}
\hline Case & Immediately after operation & 3 months & 6 months & 12 months \\
\hline 1 & $656(315)$ & $483(563)$ & $448(481)$ & $427(420)$ \\
2 & $672(471)$ & $352(748)$ & $623(730)$ & $480(740)$ \\
3 & $985(135)$ & $380(441)$ & $547(418)$ & $480(428)$ \\
4 & $833(284)$ & $629(358)$ & $441(394)$ & $420(380)$ \\
5 & $812(203)$ & $733(260)$ & $577(357)$ & $585(356)$ \\
\hline mean & $792(282)$ & $515(474)$ & $527(476)$ & $478(465)$ \\
\hline
\end{tabular}

The CT-value of the anterior part of the vertebra, in which UDPTCP was filled, and in the posterior part of the vertebra, in which the burst bones were located (in parentheses), was measured on CT performed at the indicated time points.

months, UDPTCP had completely resorbed and was replaced with the autologous bone (Figure $2 \mathrm{a}-\mathrm{h}$ ). In the other four cases, although PSs of the caudal part loosened, resorption of UDPTCP was observed at 3 postoperative months. At 6 postoperative months, progressive substitution with the autologous bone was confirmed, and at 12 postoperative months, formation of the good autologous bone was confirmed (Figure $2 \mathrm{i}-\mathrm{p}$, representing case 4 ). In all five cases, the mean $\mathrm{CT}$ values of the anterior region decreased with resorption of the artificial bone, and neared the mean CT values of the posterior autologous region by 12 postoperative months (Table 2). In 12 postoperative months, all patients were able to walk independently without pain.

\section{Discussion}

An acute vertebral burst fracture is expected to be healed by the patient's own mechanisms of fracture healing. Thus, injection of an artificial bone material in the defective bone area can effectively treat this condition. Hydroxyapatite has been used as a bone substitute in vertebroplasty ${ }^{11,}{ }^{12)}$, but it has low resorption characteristics. Alternatively, $\beta$-tricalcium phosphate has been used in humans ${ }^{13-15)}$ with good clinical outcomes. Recently, Ogose et al. compared hydroxyapatite and $\beta$-tricalcium phosphate as bone-graft substitutes after excision of bone tumors and showed that $\beta$-tricalcium phosphate was resorbed and replaced by au- 


\section{Journal of Rural Medicine}

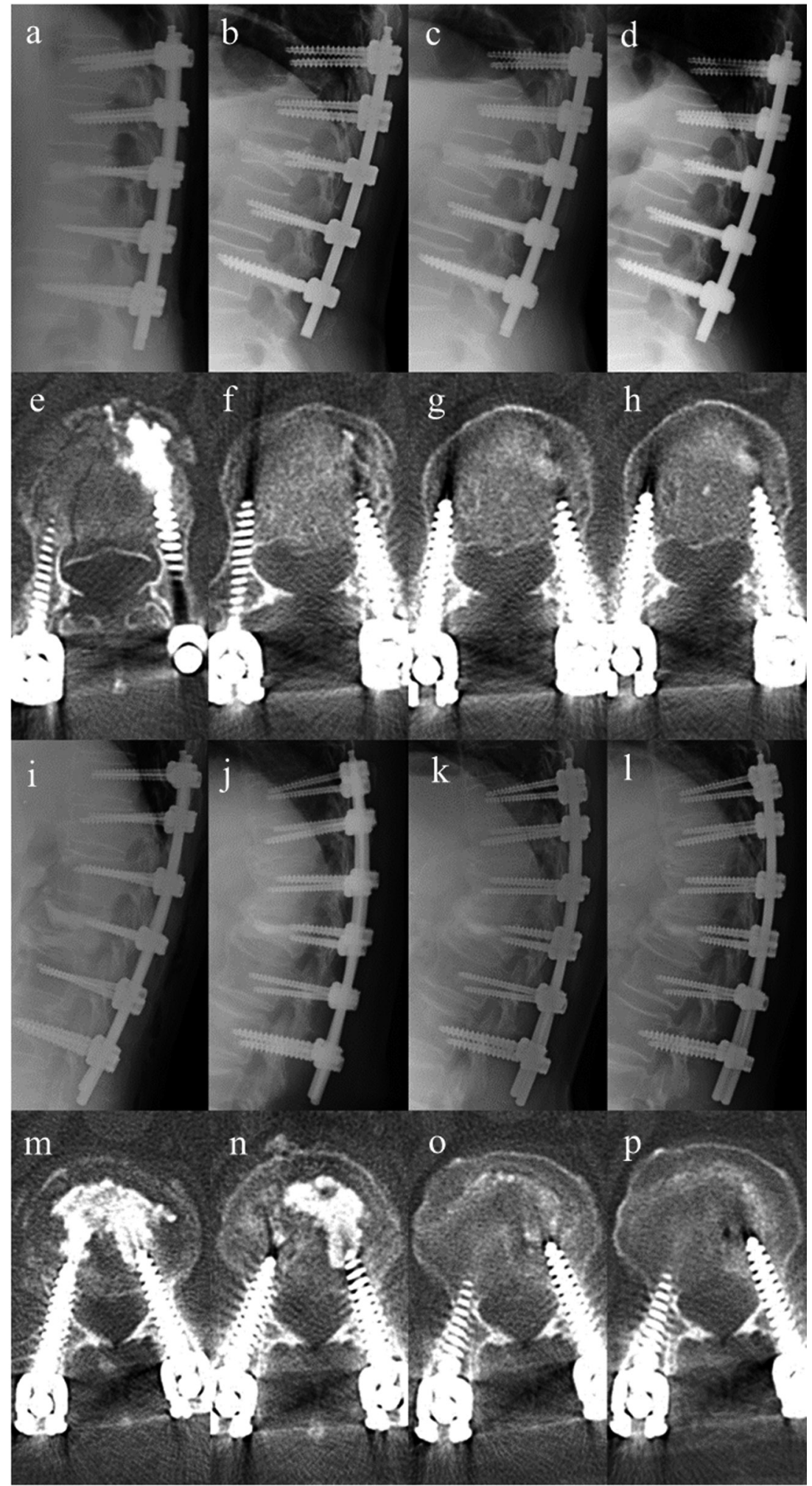

Figure 2 Postoperative time course of plain X-ray and computer tomography (CT) from two representative cases.

$\mathbf{a}-\mathbf{d}$ Plain X-ray images and $\mathbf{e}-\mathbf{h}$ CT scans of case 1. a, e immediately after the operation, $\mathbf{b}, \mathbf{f}$ at 3 postoperative months, $\mathbf{c}, \mathbf{g}$ at 6 postoperative months, and $\mathbf{d}, \mathbf{h}$ at 12 postoperative months. The pedicle screws (PS) did not loosen, and at 3 postoperative months, UDPTCP had completely resorbed and was replaced with the autologous bone. $\mathbf{i}-\mathbf{I}$ Plain X-ray images and $\mathbf{m}-\mathbf{p}$ CT scans of case 4 . i, $\mathbf{m}$ immediately after the operation, $\mathbf{j}, \mathbf{n}$ at 3 postoperative months, $\mathbf{k}, \mathbf{o}$ at 6 postoperative months, and $\mathbf{l}, \mathbf{p}$ at 12 postoperative months. Although PSs of the caudal part loosened, resorption of UDPTCP was observed at 3 postoperative months. At 6 postoperative months, progressive substitution with the autologous bone was confirmed, and at 12 postoperative months, formation of the good autologous bone was confirmed. 
tologous bone at double the rate of hydroxyapatite ${ }^{16)}$. Seto et al. reported good biocompatibility and fast resorption with uniform triple superporous $\beta$-tricalcium phosphate as bone tumor replacement ${ }^{17)}$. The newly developed UDPTCP used in the present study has a unique structure with unidirectional pores, which facilitate the ingrowth of blood vessels and bone tissue. UDPTCP has shown an exceptional balance between bioresorbability and bone substitution rates in animal models ${ }^{2,3)}$.

Our findings demonstrated that if the affected vertebrae are stabilized, UDPTCP resorption starts early after the operation, and good substitution by the autologous bone can be observed. In the elderly patients with osteoporosis, PS often loosens. This makes the affected vertebrae unstable and creates conditions adverse to fracture healing. However, in the present study, even in patients with osteoporosis whose PS loosened, the resorption of UDPTCP and substitution with the autologous bone was remarkable. Although our results may suggest that the newly developed unidirectional porous $\beta$-tricalcium phosphate shows good clinical potential as a bone-graft substitute, this report included a small number of cases. Confirmation of these results in a larger number of patients from multiple institutes is warranted.

\section{Conclusion}

In artificial bone grafting for acute vertebral burst fracture in the elderly patients, including patients with osteoporosis, the newly developed unidirectional porous $\beta$-tricalcium phosphate shows good clinical potential as a bone-graft substitute.

\section{Acknowledgment}

The authors declare that the present study was partially funded by Kuraray Co., Ltd.

\section{References}

1. Kuraray Japan Kuraray to release AFFINOS bioresorbable bone-graft substitute, available from: https://www.kuraray.co.jp/news/2015/151027, Last accessed April 17th 2019

2. Makihara T, Sakane M, Noguchi $\mathrm{H}$, et al. The balance between bone formation and material resorption in unidirectional porous $\beta$-tricalcium phosphate implanted in a rabbit tibia. Key Eng Mater 2016; 696: 177-182. [CrossRef]

3. Sakane M, Tsukanishi T, Funayama T, et al. Unidirectional porous $\beta$-Tricalcium phosphate bone substitute: examination of balance between new bone formation and absorption. Key Eng Mater 2011; 493-494: 132-134. [CrossRef]

4. Ikumi A, Funayama T, Tsukanishi T, et al. Novel unidirectional porous $\beta$-Tricalcium phosphate used as a bone substitute after excision of benign bone tumors: a case series. J Hand Surg Asian Pac Vol 2018; 23: 424-429. [Medline] [CrossRef]

5. Izawa S, Funayama T, Iwasashi M, et al. The use of unidirectional porous $\beta$-Tricalcium phosphate in surgery for calcaneal fractures: a report of four cases. Foot Ankle Online J 2017; 10: 10.3827/faoj.2017.1004.0002.

6. Kumagai $\mathrm{H}$, Abe T, Koda M, et al. Unidirectional porous $\beta$-tricalcium phosphate induces bony fusion in lateral lumbar interbody fusion. J Clin Neurosci 2019; 59: 232-235. [Medline] [CrossRef]

7. Kumagai H, Iwasashi M, Funayama T, et al. Surgical repair of acetabular fracture using unidirectional porous $\beta$-tricalcium phosphate. Case Rep Orthop 2019; 2019: 6860591 10.1155/2019/6860591. [Medline]

8. Ni WF, Huang YX, Chi YL, et al. Percutaneous pedicle screw fixation for neurologic intact thoracolumbar burst fractures. J Spinal Disord Tech 2010; 23: 530-537. [Medline] [CrossRef]

9. Uemura K, Kanamori A, Aoto K, et al. Novel unidirectional porous hydroxyapatite used as a bone substitute for open wedge high tibial osteotomy. J Mater Sci Mater Med 2014; 25: 2541-2547. [Medline] [CrossRef]

10. Tanaka T, Kumagae Y, Chazono M, et al. A novel evaluation system to monitor bone formation and $\beta$-tricalcium phosphate resorption in opening wedge high tibial osteotomy. Knee Surg Sports Traumatol Arthrosc 2015; 23: 2007-2011. [Medline] [CrossRef]

11. Takami M, Yamada H, Nohda K, et al. A minimally invasive surgery combining temporary percutaneous pedicle screw fixation without fusion and vertebroplasty with transpedicular intracorporeal hydroxyapatite blocks grafting for fresh thoracolumbar burst fractures: prospective study. Eur J Orthop Surg Traumatol 2014; 24(Suppl 1): S159-S165. [Medline] [CrossRef]

12. Nishioka K, Imae S, Kitayama M, et al. Percutaneous vertebroplasty using hydroxyapatite blocks for the treatment of vertebral body fracture. Neurol Med Chir (Tokyo) 2009; 49: 501-506. [Medline] [CrossRef]

13. Galois L, Mainard D, Delagoutte JP. Beta-tricalcium phosphate ceramic as a bone substitute in orthopaedic surgery. Int Orthop 2002; 26: 109-115. [Medline] [CrossRef]

14. Tanaka T, Kitasato S, Chazono M, et al. Use of an injectable complex of beta-tricalcium phosphate granules, hyaluronate, and fibroblast growth factor-2 on repair of unstable intertrochanteric fractures. Open Biomed Eng J 2012; 6: 98-103. [Medline] [CrossRef]

15. Tanaka T, Komaki H, Chazono M, et al. Basic research and clinical application of beta-tricalcium phosphate ( $\beta$-TCP). Morphologie 2017; 101: 164-172 [Medline] [CrossRef]

16. Ogose A, Hotta T, Kawashima H, et al. Comparison of hydroxyapatite and beta tricalcium phosphate as bone substitutes after excision of bone tumors. J Biomed Mater Res B Appl Biomater 2005; 72: 94-101. [Medline] [CrossRef]

17. Seto S, Muramatsu K, Hashimoto T, et al. A new $\beta$-tricalcium phosphate with uniform triple superporous structure as a filling material after curettage of bone tumor. Anticancer Res 2013; 33: 5075-5081. [Medline] 